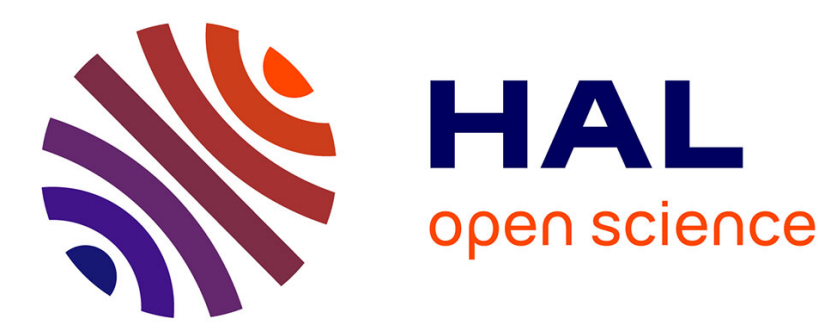

\title{
The design of post-Kyoto climate schemes: selected questions in analytical perspective \\ Roger Guesnerie
}

\section{To cite this version:}

Roger Guesnerie. The design of post-Kyoto climate schemes: selected questions in analytical perspective. 2006. halshs-00590520

\section{HAL Id: halshs-00590520 \\ https://shs.hal.science/halshs-00590520}

Preprint submitted on 3 May 2011

HAL is a multi-disciplinary open access archive for the deposit and dissemination of scientific research documents, whether they are published or not. The documents may come from teaching and research institutions in France or abroad, or from public or private research centers.
L'archive ouverte pluridisciplinaire HAL, est destinée au dépôt et à la diffusion de documents scientifiques de niveau recherche, publiés ou non, émanant des établissements d'enseignement et de recherche français ou étrangers, des laboratoires publics ou privés. 


\section{Paris-Jourdan Sciences EconomiQues \\ 48, BD JOURDAN - E.N.S. - 75014 PARIS \\ TEL. : 33(0) 143136300 - FAX : 33 (0) 143136310 \\ www.pse.ens.fr}

WORKING PAPER N²006 - 11

The design of post-Kyoto climate schemes:

selected questions in analytical perspective

Roger Guesnerie

JEL Codes : D02, D6, D7, F18, Q4,Q5

Keywords : Institutional design, fossil fuels prices, ratchet effect, quantity versus prices, tax incidence 


\title{
The design of post-Kyoto climate schemes : selected questions in analytical perspective.
}

\author{
Roger Guesnerie \\ Collège de France, Paris-Jourdan Sciences Economiques.
}

\begin{abstract}
The paper starts from a proposition of institutional design for climate policies made previously by David Bradford and labelled GPGP (Global Public good Purchase). The scheme is compared with other possible postKyoto schemes that are, or not, "Kyoto compatible". The comparison puts the emphasis on the participation issue, (free riding, ratchet effect), and on the desirable flexibility of the schemes' targets. It argues that the incidence of climate policies on the final price of fossil fuels is a key and difficult issue which has not received, untill now, the amount of required attention.

JEL : D02, D6, D7, F18, Q4,Q5

Institutional design, fossil fuels prices, ratchet effect, quantity versus prices, tax incidence

\section{Résumé:}

Ce texte part de l'examen d'une proposition d'une architecture de politique climatique due à D. Bradford et désignée sous le sigle GPGP (Global Public Good Purchase). On la compare à d'autres architectures post-Kyoto envisageables, plus ou moins "Kyoto-compatible". La comparaison met l'accent sur la participation (resquille, effet de cliquet), mais aussi sur la flexibilité souhaitable des cibles visées. L'effet indirect des politiques climatiques sur le prix final des carburants fossiles est une question essentielle et difficile qui ne semble pas avoir reçu l'attention qu'elle mérite.

JEL : D02, D6, D7, F18, Q4,Q5

Institutions, carburants fossiles, effet de cliquet, quantité contre prix, incidence fiscale.
\end{abstract}


I thank H. Tulkens for his constructive advice, O. Compte for useful criticisms on a previous version. I am particularly grateful to L. Karp and C. Philibert for their detailed comments and suggestions. I am only responsible for remaining imperfections..]Introduction ${ }^{1}$.

Climate policies raise a number of questions which have two sides. One side concerns the desirable pace of action, the other one calls for reflection on the means of action. Although they are not fully "independant", these two types of issues can be partly assessed from separate reasonings.

The first problem, the desirable pace of action, raises interesting questions, both analytically and conceptually. For example, the reflection on long run discount rates is intimately related with the assessment of the nature and extent of the uncertainty associated with the greenhouse question (see for example Guesnerie (2004)). This was the subject I had initially chosen to treat for this conference.

A second set of issues is associated with the analysis of the means of action and especially with the design of international action. This is a subject on which David Bradford has made striking propositions advocated in his paper "Improving on Kyoto: greenhouse gas Control as the Purchase of a Global Public Good" $(\text { from now GPGP })^{2}$. David had initially accepted to present these propositions at this conference. After his tragic death, the conference has been made a memorial conference in his honour. Henry Tulkens agreed with my suggestion of redefining the topics of the present lecture : it was then aimed at complementing the presentation of the paper initially proposed by David and which was read at the opening session of the conference. The lecture was hence originally conceived as an extended comment of David's propositions,

Taking note of this agenda, it should be clear, however, that the assesment of GPGP cannot be made in the vacum. In particular, it calls for comparison of the proposed scheme with the competing arrangements of the Kyoto protocol. And

\footnotetext{
${ }^{1}$ This is the revised version of the keynote lecture: "Institutional design : some analytical problems in the comparison of Post-Kyoto schemes with emphasis on GPGP" presented to the conference "the design of climate policies" held in Venice on 22-23 July 2005. The background ot the lecture and the choice of its topics are explained in the introduction.

I thank H. Tulkens for his constructive advice, O. Compte for useful criticisms on a previous version. I am particularly grateful to L. Karp and C. Philibert for their detailed comments and suggestions. I am only responsible for remaining imperfections.

${ }^{2}$ Indeed, the proposition has been circulated under two different headings, "No Cap But Trade", (NBCT), or "Global Public Good Purchase" (GPGP). Here we will stick to the second teminology.
} 
such a comparison suggests to put emphasis not on the strict version of the Kyoto protocol, but for reasons that will be explained later, on a variant of Kyoto, called Open Flexible Kyoto. Hence the paper puts comments on GPGP in the broader perspective of the comparison of GPGP with what I call "Kyoto-compatible" schemes. The comparison does not claim to be exhaustive but puts emphasis on two important dimensions of the design of climate schemes : the first one is participation and the second one is the nature of climate policy targets. Finally, enlarging the scope of the comparison of climate policies, the paper brings back a former "tax harmonisation" proposition into the picture.

The plan of the paper reflects the option taken and just explained.

- The first section proposes a brief reminder of GPGP (2-1), and then provides a first comparison of arrangements along the lines of the GPGP proposition , and of Kyoto-like arrangements, (the so-called Open, Flexible Kyoto, OFK). In this section, the comparison focuses mainly on the participation issue and successively envisages:

- The short run "participation" issue (2-2-1) which deals with the static "free riding" problem.

- The "ratchet effect" issue, which may be viewed as relating to sophisticated or dynamic "free riding". (2-2-2)

Section 2-3) summarizes the findings and then turns out making suggestions for improvement, that are particularly directed at GPGP.

- The second section turns the attention to the desirable flexibility of climate policy targets. Section 3-1-1) comes back on the conventional wisdom bearing on the comparison of "prices versus quantity", which suggests that Kyoto arrangements are too rigid. On these grounds, GPGP is shown to be more satisfactory. Section 3-1-2), however, questions the conventional wisdom from a discussion of the incidence of policies on the price of fossil fuels. Section 3-2) extends the scope of the comparison between schemes and examines how to reassess the discussion on the relative merits of "tax harmonisation" and of various "Kyoto-compatible" schemes.

Appendices 1-9 provide detailed analytical arguments. 


\section{Comparing GPGP and Kyoto like arrangements.}

\subsection{GPGP, a brief reminder.}

For the purpose of the analysis here, GPGP, which is presented in Chapter 1 of this book, is articulated as follows (Bradford (2001) :

- "Voluntary" countries provide initial contributions. For the purpose of the analysis, here, the set of contributing countries, their characteristics as well as the amount of their contributions, will usually be exogenously given.

- A Business As Usual level (from now BAU) of emissions allowances is attributed to all countries (allowances are "generous" for non contributing countries)

- An agency acquires emissions allowances i.e it buys reductions levels from the BAU level from all countries in the world, at a market price : this agency is called International Bank for Emission Allowances Acquisition. (IBEA) ${ }^{3}$

GPGP is usefully illustrated in a stylised, static and certain, world. It is assumed that there is only one-period, one single greenhouse gas and that abatment costs are certain. Formally:

Countries are indexed by $\mathrm{i} \in I$.

The costs of $q_{i}$ units of emissions abatment, measured from a Business As Usual (BAU) basis are given by a convex cost function $C_{i}$, defined for every $q_{i}$.

The utility generated in country $i$, from total reductions $Q$, is $U_{i}(Q)$, i.e $U_{i}\left(Q_{-i}+q_{i}\right)$, with $Q_{-i}=\sum_{j \neq i} q_{j}$. The function $U_{i}$ is usually assumed to be concave.

\footnotetext{
${ }^{3}$ Incidentally GPGP proposes an original system of control, which avoids measuring emissions. The Kyoto protocol relies on a measurement of emissions of participating countries. GPGP proposes a system that looks simpler : measuring imports of carbon in fossil fuels. Imports may be "extraction" from the ground within the borders or shipments across the border from another country (exports being treated from the viewpoint of the country as a negative import, cancelling the corresponding production).. Naturally, there is some discrepancy between the measured quantity and carbon emissions, but the measurement looks very simple. Accuracy of the measurement of emissions looks more of a problem. See for example, Hargrave, T., (1998), McKibbin, W. J. \& P. J. Wilcoxen, (2000)This (interesting) aspect of the proposal is only recalled here for memory and is not discussed further. For the sake of the comparison attempted here, import allowances are not distinguished from emissions allowances.
} 
There are two groups of countries. They are called respectively (and metaphorically $^{4}$ ) Annex B, $(i \in B)$, where $d U_{i} / d Q>0$, in the whole range under consideration and non Annex B countries $(i \in N B)$ that are supposed to be unconcerned ${ }^{5}$ by the policy, i.e to be associated with $d U_{i} / d Q=0, \forall Q,\left(U_{i}=C s t e(=0)\right)$.

Let us now consider a GPGP equilibrium, associated with exogenously fixed contributions $\overline{T_{i}} . i \in B$

Definition 1. A GPGP equilibrium, associated with fixed contributions $\overline{T_{i}}, i \in B$, consists of abatments $\left(q_{i}^{*} \geq \overline{\overline{q_{i}}}, i \in B, q_{i}^{*} \geq 0, i \in N B\right.$ ), a positive "carbon price" $t^{*}$, such that :

1. $q_{i}^{*}$ is a solution of $\operatorname{Max}\left\{U_{i}\left(Q_{-i}^{*}+q_{i}\right)+t^{*}\left(q_{i}-\overline{\overline{q_{i}}}\right)-C_{i}\left(q_{i}\right)\right\}, q_{i} \geq \overline{\overline{q_{i}}}, i \in B$, with $Q_{-i}^{*}=\sum_{j \neq i} q_{j}^{*}$

2. $q_{i}^{*}$ is a solution of $\operatorname{Max}\left\{t^{*} q_{i}-C_{i}\left(q_{i}\right)\right\}, i \in N B$.

3. $\sum_{i \in B} t^{*}\left(q_{i}^{*}-\overline{\overline{q_{i}}}\right)+\sum_{i \in N B} t^{*} q_{i}^{*}=\sum_{i \in B} \overline{T_{i}}$

The GPGP equilibrium is said to be individually rational iif

4. : $\left\{U_{i}\left(Q^{*}\right)+t^{*}\left(q_{i}^{*}-\overline{\overline{q_{i}}}\right)-C_{i}\left(q_{i}^{*}\right)-\bar{T}_{i}\right\}>U_{i}\left(\sum_{i} \overline{q_{i}}\right)-C_{i}\left(\overline{q_{i}}\right)$, with $Q^{*}=\sum_{i} q_{i}^{*}, i \in$ $B$, and where $\overline{q_{i}}$ is an a priori given reference level.

The interpretation is simple and reflects, in the given context, the above unformal presentation of the scheme :

$B$-countries contribute the - exogenously - fixed amount $\overline{T_{i}} ; t^{*}$ is the carbon price paid by the central agency; the BAU levels of abatments are $\overline{\overline{q_{i}}}$, in B-countries and zero in NB countries; abatments of country $i \in B$ equal $\left(\operatorname{Max}\left(0, q_{i}^{*}-\overline{\overline{q_{i}}}\right)\right.$ or equal $q_{i}^{*}, i \in N B$,so that the sum of payments, associated with abatments beyond the BAU level, is given by the left-hand side of equation 3 and equals the right hand side $\sum_{i \in B} \overline{T_{i}}$.

Conditions 1 and 2 define the optimal decision respectively of B and NB countries.

The viability condition, equivalently an individual rationality condition, expresses the fact that a $B$-country is better off than in an (the) initial reference

\footnotetext{
${ }^{4}$ Metaphorically, because the terminology suggests that the GPGP contributing countries would be the Annex B countries of the Kyoto protocol.

${ }^{5}$ This is a too extreme assumption but a reasonable modelling option to separate B and non B countries.
} 
situation. This condition is necessarity satisfied for a $N B$-country, but not necessarily for a $B$-country if its initial contribution (or its ${ }^{6} \overline{\overline{q_{i}}}$ ) $\overline{T_{i}}$ is too high. Here as in the Appendix, we assume that the reference situation is an anterior non cooperative Nash equilibrium of abatments ${ }^{7}$. In the remaining, we always assume that $\overline{\overline{q_{i}}}={\overline{q_{i}}}^{8}$.

Our evaluation of GPGP and its comparison with Kyoto like arrangements will first consider the so called free riding issue.

\subsection{Participation.}

\subsection{1. "Standard" free riding.}

There have been many reflections on the stability of climate policy arrangements and this conference has a number of contributions on this subject.

Indeed, the Kyoto arrangements are most vulnerable to some form of "free riding". A cooperative policy can be expected to be stable only in a weak sense: Carraro (1999) reviews existing results and a number of papers in this conference are concerned with this issue (in particular H. Tulkens'one) ${ }^{9}$.

One main argument for GPGP is that it solves, or at least powerfully helps solving, one problem coming under the heading of "free riding", i.e the participation problem. If the BAU cap is computed correctly, then it is in the unambiguous interest of a NB-country to participate in the abatment process : it cannot lose but, by selling allowances, only win. As we assume in the definition that the arrangement is viable for B-countries (this is the viability constraint 4), all countries are willing to participate. Clearly, this is true in our stylised model, but would remain true, in an uncertain world, only if the cap were made contingent on

\footnotetext{
${ }^{6}$ We note that any change in $\overline{\overline{q_{i}}}$ can be matched by a change of $\overline{T_{i}}$, that leaves the equilibrium unchanged. Viewing formally the contribution as a money contribution plus an abatment contribution, allows, without loss of generality, to take the quota associated with a $B$-country, $\overline{\overline{q_{i}}}$, to be 0 .Naturally, the cost function would have to be redefined accordingly.

${ }^{7}$ Indeed, the fact that the BAU levels are taken to be zero for NB countries and non zero for B countries, is supposed to reflect the fact that in the anterior Nash equilibrium, (defined in Appendix 2) B countries would abate when NB would not.

${ }^{8}$ Which, from the two previous footnotes, might be taken equal to zero, a convention which would however affect the interpretation of $C_{i}$ and in particular of $C_{i}(0)$.

${ }^{9}$ Very briefly, one may note that there are some forces for cohesion in the sense that "free riding", for most formal definitions of the word, is unlikely to be a "dominant strategy" for any actor, but these cohesion effects are limited in the sense that defecting from the arrangement, at least if one assumes that the others will stay, is most often an optimal strategy.
} 
enough verifiable events. Hence, as David Bradford suggested, the computation of contingent BAU levels should be enough accurate and if not, enough "generous".

Hence, although contribution to the the financing is optional and made on voluntary basis, the design would trigger self advantageous participation of all nations in the abatment scheme.

This is apparently a decisive advantage: a scheme that leaves out of the arrangement a significant part of the world suffers from serious drawbacks. The most significant one is visualised (and possibly caricatured) from the curve below (Figure 1): on the horizontal axis is the effort of the climate coalition (measured for example by the carbon price), on the vertical axis is the reduction of the world emissions.

\section{Figure 1}

Figure 1 depicts a humped shaped curve: at a low level of effort of the coalition, the emissions decrease when the effort increases, but the amount of reductions may go to a maximum. The reason is that polluting industries of the coalition lose market shares and/or migrate outside and this happens more and more whenever the effort level of the coalition increases. Indeed, under some assumptions on the "out of the coalition" standards, reductions might further decrease with the effort level, associating possibly a higher effort level with lower performance. A still sketchy but more formal presentation of the argument is provided in Appendix 1. It puts emphasis on the elasticity of substitution beteween "de-carbonated" and "carbonated" goods. Although, given present estimates, the phenomenon seems unlikely in the aggregate, unless the climate coalition level of effort becomes very high, a bold empirical assessment of the parameters of the formal analysis would suggest that the phenomenon ("carbon leakage" over 100 per cent) is plausible at the industry level : increasing the effort in virtuous countries might increase the world emissions within a given industry, when the coalition level of effort becomes significant (for an empirical assessment, see Demailly-Quirion in this volume).

One will scrutinize below the nature of the advantage of GPGP scheme over Kyoto like schemes, (from now K). To do that, it is useful to define a Flexible Kyoto arrangement, (flexible meaning that quotas are tradable on an international market for permits, as they are in the actual treaty).

Definition 2. a Flexible Kyoto equilibrium consists of (exogenously given) quotas $\widehat{q_{i}}, i \in B$, abatments $\left(q_{i}^{* \prime}\right), i \in B$, a positive "carbon price" $t^{*}$, such that :

1. $q_{i}^{* \prime}$ is a solution of $\operatorname{Max}\left\{t^{*}\left(q_{i}-\widehat{q}_{i}\right)-C_{i}\left(q_{i}\right)\right\}, i \in B$, 


\section{2. $\sum_{i \in B} q_{i}^{* \prime}=\sum_{i \in B} \widehat{q_{i}}=Q^{* \prime}$}

It is individually viable if

$$
\text { 3. } \left.\left\{U_{i}\left(Q^{* \prime}\right)\right)+t^{*}\left(q_{i}^{* \prime}-\widehat{q}_{i}\right)-C_{i}\left(q_{i}^{* \prime}\right)\right\}>U_{i}\left(\sum_{i} \overline{q_{i}}\right)-C_{i}\left(\overline{q_{i}}\right) \text {. }
$$

In a flexible Kyoto equilibrium, the sum of abatments equals the sum of initially attributed quotas, (NB-countries are not concerned), and is taken as given in the individual optimisation that takes into account the cost and the market price of abatments (1). But quotas are traded, and the equilibrium price of the market for permits is $t^{*}$. Note that in the definition, quotas are fixed, even if they

may have been ex-ante bargained in order to vary the burden across participating nations ${ }^{10}$.

But, naturally such an FK scheme does not solve the participation problem of Developing Countries (this is clearly true in our stylised model, where NBcountries are not participating $)^{11}$. Given the failure of FK to trigger participation of a part of the world in which emissions are likely to become significant, the scheme is then likely to be potentially much less effective than a GPGP scheme: this participation argument is clearly at the heart of D. Bradford's proposition

However, I would like to suggest that it seems fair to compare GPGP not necessarily with the actual Kyoto arrangement, but with variants of Kyoto that would mimick the GPGP solution to the so-called participation problem.

To this aim, one variant of Kyoto, denoted (OFK), Open Flexible Kyoto, is now proposed : those countries which are non contributing in the GPGP scheme would be given, within the FOK scheme, the same BAU targets they have in GPGP and would be induced to participate in a rather similar way. Indeed, such targets, called nonbinding targets, (or in a slightly different way "no-lose" or

\footnotetext{
${ }^{10}$ Contrarily to "real" Kyoto arrangements, where the quotas are fixed in reference with the countries'levels of emissions at a given year (1990), with little possibility of bargaining, (but on the reference year itself) around this reference arrangement.

It seems however that increasing the number of degrees of freedom in the arrangement, by playing in a more flexible way on the repartition of national quotas might favour stability in some sense, although it might make the negotiations trickier. As an illustration of this idea, the ex-post favourable treatment of Russia, turned out to be conducive to (incomplete)stability of the Kyoto arrangements. However this was obtained by "luck" rather than by the design of the negociations design.

${ }^{11}$ In the real world, NB-countries are induced to participate via the CDM mechanisms : this seems however to provide only limited incentives.
} 
"one way" targets) have been proposed as a way to induce participation of the Developing Countries in the Kyoto scheme (in particular by Philibert (2000) or Philibert and Pershing (2001)).

Here is a formal definition of OFK :

Definition 3. an Open Flexible Kyoto equilibrium consists of (exogenous) quotas $\widehat{q}_{i}, i \in B$,abatments $\left(q_{i}^{* *}\right), i \in I$, a positive "carbon price" $t^{* *}$, such that :

1. $q_{i}^{* *}$ is a solution of $\operatorname{Max}\left\{t^{* *}\left(q_{i}-\widehat{q}_{i}\right)-C_{i}\left(q_{i}\right)\right\}, i \in B$,

2. $q_{i}^{* *}$ is a solution of $\operatorname{Max}\left\{t^{* *} q_{i}-C_{i}\left(q_{i}\right)\right\}, i \in N B$,

3. $Q^{* *}=\sum_{i} q_{i}^{* *}=\sum_{i \in B} q_{i}^{* *}+\sum_{i \in N B} q_{i}^{* *}=\sum_{i \in B} \widehat{q}_{i}$

4. It is individually viable if $\forall i \in B:\left\{U_{i}\left(Q^{* *}\right)+t^{* *}\left(q_{i}^{* *}-\widehat{q}_{i}\right)-C_{i}\left(q_{i}^{* *}\right)\right\}>$ $U_{i}\left(\sum_{i} \overline{q_{i}}\right)-C_{i}\left(\overline{q_{i}}\right)$, with $Q^{* *}=\sum_{i} q_{i}^{* *}$.

In an Open Flexible Equilibrium, the environnemental performance is still given by the quotas of the Annex B countries, but non Annex B countries, which are given non binding commitments, participate, and, as in a GPGP scheme, benefit from participation, while decreasing the cost of Annex B countries.

Appendix 2 repeats the formal definitions of the just evoked schemes GPGP, OFK, while introducing some others (Nash Equilibrium) needed for a comprehensive analysis, always within the stylized framework of a simple world without uncertainty. It is shown that the definitions are not empty (in particular, for fixed contributions, there exists a unique GPGP equilibrium, which is convenient for the analysis, although not obvious).

The analysis suggests (proposition 6) that, if one forgets the viability constraints (4), the abatment targets attainable through GPGP schemes, when the contributions are varied, are identical to the abatment targets attainable in an OFK scheme, when the quotas are varied ${ }^{12}$.

The analysis also suggests, although it is not always true, that the contributing countries, let us call them annex B countries, using the Kyoto vocabulary, are likely to be better off in a GPGP scheme when compared with an OFK scheme that leads to the same ecological performance. Hence participation constraints for B-countries are more likely to be satisfied with GPGP.

\footnotetext{
${ }^{12}$ This may justify to view GPGP, as in Guesnerie (2003), as "Kyoto-compatible" !
} 
This may look surprising ${ }^{13}$. The reason is that in a GPGP scheme annexB countries, when faced with some given carbon price, tend to have a more ambitious abatment policy than when they face the same carbon price in an OFK scheme; taking that into account, they may pay a cheaper bill in order to attract non Annex B countries (Corollary7).

Note that the so suggested superiority of GPGP for ensuring participation is probably weak : I conjecture that it decreases when the size of Annex B increases. Also, the property does not necessarily imply that coalitional stability, in a more demanding sense, would be more likely with GPGP, i.e that the negotiation between Annex B countries would be easier under GPGP. We have nothing to say here on the more general issue that comes under the heading of "coalitional stability".

Note also, as argued by David Bradford, that one advantage of GPGP, for real world negotiations, is transparency of the costs, when transparency of the costs of OFK is only guaranteed in a world without uncertainty ${ }^{14}$. Hence, actual differences in stability of arrangements between GPGP and OFK may reflect real effects, as the ones just stressed in a certainty context, but also those due to uncertainty, notwithstanding possible "framing" effects (the fact that two equivalent games are played differently according to a priori insignificant differences of formulation).

At this stage, the advantage of GPGP over the actual Kyoto (K) arrangements, in terms of participation, is clear. But it is not decisive, in the sense that variants of Kyoto may mimick one central feature of GPGP : voluntary costly participation could be in principle triggered, as well as voluntary non costly participation, by adopting an Open Flexible Kyoto (OFK). And, as just argued, and from the analysis presented in the Appendix, the comparison between GPGP and OFK, while suggesting an advantage for GPGP, remains at this stage somewhat unconclusive.

\subsubsection{The ratchet effect.}

One should however stress that participation decision of the countries that are supposed to join is less obvious than suggested by the previous analysis, a fact of which D.Bradford was fully aware of. Indeed, the previous argument and the

\footnotetext{
${ }^{13}$ Particularly, in view of the fact that the latter might induce Pareto optimality when the former cannot.

${ }^{14}$ For OFK, without uncertainty, the outcome is in principle "transparent".
} 
stylised one made in David Bradford's paper take place in a static world. In a dynamic world, the fixation of initial BAU quotas will be followed, next period, by the fixation of next BAU quotas. The scheme will face what is known to be a "ratchet effect". The ratchet effect has plagued the performance of the Soviet economy, where firms were reluctant to seize present bonuses under the expectation that current quotas would be raised to-morrow as a consequence. Asymmetric information between the Center and the firms, and absence of commitment from the Center, lie at the heart of the ratchet effect, and optimal bonuses may be designed, taking into account the asymmetry of information and non-commitment. (see Freixas-Guesnerie-Tirole (1985)).

In the GPGP scheme, the ratchet effect takes a simple form : a country, when considering selling its allowances, should take into account not only the benefits from its present sale, but also, possibly the loss due to the effect of its decision on its next amount of BAU allowances. It is easy to imagine circumstances where the participation decision, particularly of non contributing countries, (non annex B, in our terminology) would be postponed. Even if it were not the case, the actual incentives to reduce emissions would be lower than the apparent monetary incentives ( $\mathrm{x}$ dollars per ton of carbon on the market for allowances).

Hence, the main virtue of GPGP i.e that it solves the Developing Countries participation problem, is less convincing than it looks. Naturally, the same remarks apply to the related devices that would sustain an OFK scheme (non binding constraints, one-sided constraints) ${ }^{15}$.

The remedy to the ratchet effect, whether we are in an OFK scheme, or a GPGP scheme has a name: (long term) commitment. Strict commitment would mean that the BAU allowances of GPGP are fixed at the outset for a very long period of time. But the number of contingencies to be taken into account in the determination of allowances would become unrealistically high. An (partial) alternative would be to agree on long run targets, which then would likely be somewhat uniform between countries, (and then might refer to per capita emissions, may be corrected by some production indices and some climate damages exposure). This would not suppress opportunistic considerations in the abatment decisions but would limit their scope.

Again, one may think that the ratchet effect plagues, in somewhat similar ways, GPGP and Kyoto schemes. Solutions are similar under both types of arrange-

\footnotetext{
${ }^{15}$ Also, in the present Kyoto protocol, the ratchet effect is likely to appear at the renegotiation stage, in spite of some existing dispositions that penalize non attainment of the objectives. Countries that have performed poorly are likely to take this as a case for lower future objectives.
} 
ments but presently are of dubious feasibility.

\subsection{Suggestions for improvement and first conclusions.}

Let us sum up. David Bradford proposition's revives an old procedure for financing public goods, voluntary contribution; it does that while showing that countries that are not willing to finance the scheme might be given allowances that induce them to participate in the implementation of the climate policy. We have just argued that the scheme favours participation, in a way that makes it superior to the present Kyoto arrangements, but in a way that might be mimicked by variants of the Kyoto scheme. However, both an improved Kyoto scheme and GPGP scheme are likely to be plagued by ratchet-like effects.

Taxation is another way, which historically has been most successful to finance public. Let us suggest that in a GPGP framework, the financing rules might be changed to rely (partly) on an emission tax, paid at each period by every country and based on the amount of its previous period emissions. In principle, the taxation scheme would remain combined with a voluntary contribution, (the a priori weight given to the taxation component of the resources of the agency should be thought carefully). Hence, every country, would have (part of) its contribution, at least in future periods, determined by a tax on its emissions of the previous period.

One will object that taxation is associated with the "regalian" power of a Government and there is no such thing as a world Government, to-day and in a predictable future. However, in this variant of the scheme, voluntary participation might still be triggered, probably at levels of total contributions similar to those of the initial GPGP scheme.

The idea is simple and may be more conveniently illustrated, by introducing formally a GPGPPT scheme (Global Public Good Purchase Plus Taxation).

Definition 4. A GPGPPT equilibrium, associated with 'exogenous) contributions $\overline{T_{i}} . i \in B$, consists of abatments $\left(q_{i}^{*} \geq \overline{\overline{q_{i}}}, i \in B, q_{i}^{*}>0, i \in N B\right)$, a positive "carbon price" $t^{*}$, such that :

1. $q_{i}^{*}$ is a solution of $\operatorname{Max}\left\{U_{i}\left(Q_{-i}^{*}+q_{i}\right)+t^{*}\left(q_{i}-\overline{\overline{q_{i}}}\right)-C_{i}\left(q_{i}\right)\right\}, q_{i} \geq \overline{\overline{q_{i}}}, i \in B$, with $Q_{-i}^{*}=\sum_{j \neq i} q_{j}^{*}$

2. $q_{i}^{*}$ is a solution of $\operatorname{Max}\left\{t^{*} q_{i}-C_{i}\left(q_{i}\right)\right\}, i \in N B$. 
3. $\overline{T_{i}}=\bar{t} E_{i}^{-1}+e_{i}$, wheret is a tax rate and $E_{i}^{-1}$ is the emissions of country $i$ at period -1 .

4. $\sum_{i \in B} t^{*}\left(q_{i}^{*}-\overline{\overline{q_{i}}}\right)+\sum_{i \in N B} t^{*} q_{i}^{*}=\sum_{i \in B} \overline{T_{i}}$

It is straightforward to check that in the short run, (see Appendix 4) the initial voluntary contributors in the GPGPPT scheme may be given the same burden than in the original GPGP scheme.

Going further, making this tax non linear would make sense. In particular, introducing an exemption level would be in line with leaving Developing Countries temporarily out of the business of financing and indeed would be part of the rationale for the separation between B and Non B countries.

Let us stress two virtues of the mix of voluntary contribution and taxation involved int the suggested amendment.

First, in such a modified GPGP framework, the tax would both contribute to provide the financing means and would have a "leverage effect" on incentives: assume that, in a steady state, the price of carbon on the market managed by the IBEA agency is y dollars per ton and the (marginally flat) tax on carbon emissions is $\mathrm{x}$ dollars per ton, then, forgetting about discounting, the incentives to reduce emissions of one unit in the present period is $\mathrm{x}+\mathrm{y}$, (instead of $\mathrm{y}$ ).

Second, and consequently, the system would hence be likely to considerably limit the ratchet effect. This would be the case, in particular, of countries that currently do not contribute and are not taxed, since they would have to consider how the present effort contributes to determine some future taxation burden. The curious reader will note that the somewhat hidden hypothesis that explains the argued increase in performance of the new scheme is not that it involves some "quantity commitment" on the future, but that it sustains some kind of a "procedure commitment" i.e the fact that the taxation of past emissions will be on the negociation table at the next stage of the game.

Whether this is a politically realistic post-Kyoto scheme remains unsure. It seems however to provide an arrangement possibly superior to the initial GPGP and to OFK (although OFK might be amended with a tax arrangement analogous to the one suggested here and serving to finance a minimum carbon price..).

Introducing taxation suggests to extend the discussion on possible climate policies schemes, and to compare either the initial GPGP scheme, or its (hopefully) improved variant just suggested (Global Public Good Purchase Plus Taxation, 
GPGPPT), or OFK with another possible scheme, that was suggested ${ }^{16}$ at an early stage of the climate negotiations, and that was labeled "harmonized taxation". Harmonised taxation is often viewed as a prototype 'price" policy, opposed to the Kyoto-like "quantity" policy. We will recall this distinction in the next section, while assessing the intermediate status of GPGP. As a prerequisite to the general discussion, we shall ask whether we should stick, in the comparison of prices versus quantity policy, to the conventional wisdom.

\section{Flexible or rigid targets : GPGP, Kyoto-like schemes and \\ Tax harmonisation.}

\subsection{Prices versus quantities:}

\subsection{1. the standard viewpoint.}

The Kyoto protocol implements a quantity policy, in the sense that it sets global objectives in terms of emissions for the participating partners. National performances may differ from national quotas, since these quotas can be exchanged on an international market that allows partners to sell or to buy emissions allowances. But the total authorized amount of emissions is left unchanged. This basic option of the Kyoto protocol has been criticized on the grounds that it is not enough flexible, given the present uncertainty on the costs of abatment. It leads to too little reduction, if it turns out that the (marginal) cost of abatment is smaller than expected; it leads to too much reduction in the contrary case. Hence, the performance of a quantity policy is suboptimal in the presence of uncertainty.

A price mechanism, (implicitly associated with a uniform tax on carbon emissions) also leads to a suboptimal performance, although in a different way: it introduces a discrepancy with the optimal effort that increases with the slope of the marginal benefit curve. The comparison between the two policies, price and quantity, which are in general both non-optimal, is usually made along the lines of argument just briefly suggested and initially stressed by Weitzman (1974).

The transposition of the argument to the greenhouse problem requires making assumptions on the shape of the marginal benefit curve: it is in general assumed to be flat on the grounds that the amount of reductions under consideration is small compared to the stock of carbon dioxide in the atmosphere. Under

\footnotetext{
${ }^{16}$ It was the suggested by the Europeans and rejected by the US. Positions have now switched to some extent..
} 
this assumption, a price policy would be superior to a quantity policy. The argument of the superiority of the so-called "price policy" over the "quantity policy", in a climate context, has been revisisted and confirmed in a dynamic framework both analytically sophisticated and with operationally plausible calibrations (see the work of Newell-Pizer (2000) and Pizer(2001)). A number of objections to the approach (and hence to the conclusion) have been made (see Karp-Hoel (2001), (2002), Karp-Zhang(2005), Victor(2001)). They will not be discussed here where emphasis will be put later on different objections. At this stage, one may note here that the so-called price policy, the superiority of which is claimed, should aim not only at partial tax harmonisation, (a - small - additional carbon tax equal for all countries). Rather, it should likely lead to significant tax harmonisation, (a significant carbon tax that would wipe out the present huge differences in total carbon taxation between countries). One may wonder whether it should not possibly aim at a more ambitious goal, something like stabilising the total price of carbon (which reflects not only the carbon tax but also the price of the carbon implicit to the price of the fossil fuel where it comes from). We shall come back on that later.

Here, let us only note that along the lines of the standard price quantity argument, a GPGP performs better than a $K$ scheme. The reason, developed in Appendix 6, is that in the price-quantity space, the outcome of a GPGP scheme obtains from the intersection of an hyperbola (corresponding to a fixed expenditure - product of price by quantity - ) and of the marginal cost curve. It then allows less quantity abatment fluctuations than would be optimal but more fluctuations than triggered by a pure quantity policy, (with comparable expected expenditures).

\subsubsection{Prices versus quantities : should we stick to the conventional wisdom?}

One will sketch here a critical review of the standard assessment, (conventional wisdom) which is, on several points, tentative but which puts the comparison of quantity policies (Kyoto), price policy (tax and price harmonisation) and mixed price-quantity policies (GPGP) in a better perspective.

First, there are technical objections to the leading defence of the standard argument, which do not seem to have been stressed the earlier literature and in the previously quoted papers. One of them relates with time-aggregation and the separability of costs: a sequential model in which the marginal cost curve in 
each period is increasing says that the first action at time $t+1$ is (much) less costly than the last action at time $t$ and that these costs are independant.This modelling artefact may bias the argument against quantity policies.

- Second, the analysis of the outcome of a pure taxation policy, without quantity constraints, should take into account the reactions of the prices of fossil fuels to the taxation scheme. The issue is delicate, for several reasons, among which one may single out two. First, the short run and the long run incidence effects have to be disentangled, second, the pricing of fossil fuels has oligopolistic dimensions that make it actually more difficult to assess than in the reference competitive model.

Let us recall the basic insight of the static, competitive model of the exploitation of an exhaustible resource : there, a tax has no effect on the total price and only transfers the rent from the producers to the taxing authority. This key static insight has dynamic counterparts. Tax increases trigger offsetting movements of producers prices (although only very special time patterns of tax changes will leave final prices and quantities unchanged); taxes also help here transferring the rent from the owners of the exhaustible resource to the consuming countries; finally, it is only an appropriate modulation of taxes through time that allows to delay the exhaustion time of the resource. (See Appendix 8). Although there has been in the past a significant amount of work on the price of fossil fuels (see the survey of Karp-Newbery(1993)), the climate policy dimension of the problem does not seem to have attracted much specific work. An exception is ChakrovortyMagné-Moreaux (2003), Magné-Moreaux (2002)): although these papers take into account neither uncertainty nor oligopolistic pricing, they convey messages that seem most relevant for the design discussion and hence deserve more scrutiny.

\subsection{Tax harmonisation versus Kyoto-compatible schemes.}

Harmonised taxation has been suggested as a superior arrangement when compared to Kyoto-like schemes. We will sketch a reassesment of the issue while including GPGP and OFK into the picture.

- What is meant by tax harmonisation?: Section 3-1-1 distinguishes partial tax harmonisation, (an additional carbon tax equal for all countries), full tax harmonisation, (a carbon tax roughly uniform when the present situation involves huge differences between countries), or, may be, a stabilized and planned total price of carbon $^{17}$.

\footnotetext{
${ }^{17}$ Which, again, is the sum of the carbon tax plus the price of the carbon implicit to the price
} 
The formal definition of an harmonized tax equilibrium, in the simple framework considered in Appendix 2, assures that the carbon price can be controlled, and would be generally associated with full tax harmonisation, although one will challenge this interpretation later.

Definition 5. An harmonised Tax equilibrium consists of abatments $\left(q_{i}^{00}\right), i \in I$, a positive "carbon tax" or "carbon price" $t^{00}$, such that :

1. $q_{i}^{00}$ is a solution of $\operatorname{Min}\left\{t^{00}\left(q_{i}\right)-C_{i}\left(q_{i}\right)\right\}, i \in I$,

2. $Q^{00}=\sum_{i} q_{i}^{00}=\sum_{i} R_{i}\left(t^{00}\right)$, where $R_{i}(t)$ is the solution of $\operatorname{Max}\left\{t q_{i}-C_{i}\left(q_{i}\right)\right\}$

3. The welfare of country $i$ is $\left\{U_{i}\left(Q^{00}\right)-C_{i}\left(R_{i}\left(t^{00}\right)\right)\right\}$

- The definition calls for several comments.

a. Tax harmonisation does not leave any degree of freedom in the repartition of burdens ( as analysed in D. Bradford's (2002) appendix). Its outcome can be mimicked, again in the simple world of Appendix 2 considered here, by a Kyoto-like arrangement where quotas are such that there is no trade on the world permit market (see Appendix 7).

b. Placing severe limits on the income transfers that are possible may be viewed as an advantage when income transfers from developed countries to developing countries are viewed with suspicion ${ }^{18}$. There is an actual additional advantage: since there is no strict connection between present and future targets, an harmonised taxation scheme is in principle less vulnerable to the form of the ratchet effect described above.

c. The impossibility of transfers seems however to have serious inconveniences : income transfers give more flexibility in the search of a stable (ambitious) arrangement; in particular, as it is the case in our simple model, unanimous voluntary participation is likely to be trigerred only at lower levels of effort.

- The complex relationship between prices of fossil fuels and taxes, suggest that full tax harmonisation strictly speaking would leave rather uncertain the final price of fossil fuels. Hence, the above model would really describe a

of the fossil fuel where it comes from.

${ }^{18}$ Such a suspicion seemed to be implicit to the US position in the negotiations of the Kyoto protocol. 
carbon price control policy and not an harmonised taxation situation ${ }^{19}$. In fact, the quantity performance associated with the harmonised taxation policy may be grossly suboptimal, when compared to the carbon price control policy imbedded in the above definition.

There are multiple reasons for such a suboptimal performance. They may be analysed in a simplistic world of a single exhaustible resource with competitive owners with perfect foresight. First, prices responses may partly offset the effect of taxes. Second, one may be skeptical vis à vis the long run outcome of tax harmonisation (or even pure price policies), that are not committed at the outset : the time profile of taxes should be announced in a credible way at the outset, unless the final price reaction will be difficult to assess. Third, the quantity reactions of providers of the exhaustible resources is plagued by "eductive instability" 20 etc. And the real world uncertainty of the outcome is probably greater than what the simplistic analysis alluded to before suggests.

- Appendix 9 shows, graphically, why, when the above considerations are taken into account, the ranking of a pure Kyoto quantity policy, of a GPGP arrangement or a tax harmonisation with uncertain effects, is ambiguous, even when the marginal benefit curve is flat.

On the whole, the reassessment of the price versus quantity debate, only attempted here, gives more credence to a pure quantity policies associated with Kyoto schemes and introduces another and yet unexplored dimension of comparison between GPGP and OFK, a dimension which may weaken the case for GPGP . Further and new reflection on the effects of carbon tax schemes on fossil fuels pricing should be most welcomed..

\section{Conclusion}

This paper has compared GPGP and Kyoto like schemes.

It has been argued that the advantage of GPGP, as a powerful device for triggering universal participation, can be mimicked by a variant of Kyoto, called

\footnotetext{
${ }^{19}$ The interpretation of our model of GPGP is subject to a similar objection, althufh the OFK modelling is not.

${ }^{20}$ For an "eductive" viewpoint on the conditions of expectational coordination, see Guesnerie (2005). The fact that price coordination is in this context strongly "eductively unstable", is straigthforward in the simplest models suggested above.
} 
OFK. The comparison between OFK and GPGP is partly unconclusive, although the latter has three strong points. First, it seems (slightly) superior for triggering participation; second it can be modified to include some kind of emissions taxation in a way that would attenuate the ratchet effect; third it determines more flexible targets than Kyoto schemes and is hence less exposed to the standard criticism made to quantity policy.

However, it has been argued in the last Section, that the conventional wisdom on the relative virtues of price versus quantity has to be re-examined in depth, in order to take into account the reaction of the price of fossil fuels to carbon taxes. When this dimension is taken into account, the merits of harmonized taxation will have to be reassessed downwards, and the evaluation of GPGP and OFK, in terms of target flexibility, may be significantly affected, reopening the debate on their the relative merits.

\section{Appendix1: Effort level in Annex B and global reduc- tions.}

Let assume that the effort level in Annex B countries is measured by the "normalized" price of carbon, denoted $t$, so that the price of the carbon intensive good is $p+t$, the level of reductions per unit of the carbon intensive good is $(\partial C)^{-1}(t)$, so that the level of reductions is $D(.)(\partial C)^{-1}(t)$, where $D($.$) is the demand for the$ good.

In non annex $\mathrm{B}$ countries, the price is $p$, but no reduction action is implemented, and there is at the outset an excess emission of $u$ per unit of production.

Calling respectively $D(B, .,$.$) and D(N B, .,$.$) the demand functions for the$ goods produced in the two areas, the total level of reductions, as a function of $t$, is $: \Delta(t)=I[D(B, p, p)-D(B, p+t, p)]+(\partial C)^{-1}(t) D(B, p+t, p)-(I+u-$ $\epsilon)[D(N B, p+t, p))-D(N B, p, p)]$

where $I$ is the initial carbon content of B-goods and where $\epsilon$ may (here exogenously) take into account the fact that the new demand in Annex B countries is met between firms using the old nonAnnex B technology and migrating firm using the initial Annex B technology.

One has $(d \Delta / d t)=-\partial_{1} D(B, .,).\left\{I-(\partial C)^{-1}(t)\right\}+\left(\left(\partial C^{\prime}\right)^{-1}(t)\right) D(B, .,)-.(I+$ $u-\epsilon)\left[\partial_{1} D(N B, .,).\right]$

The shape of $\Delta(t)$ depends on the form of the demand function, but is as suggested in the text under a number of circumstances. For example, with CES 
demand functions, when the elasticity of sustitution between the de-carbonated good and the carbonated good is greater than one, the effect of an increase in the carbon tax in the B-countries on total emissions becomes ambiguous : the two first (positive) terms may become dominated by the last term (which is negative). Note however than the required elasticity of substitution is more plausible at the industry level (steel, for example), than at the aggregate level. It is left to the reader to illustrate the phenomenon from his own crude empirical estimates at the industry level, (steel..).

\section{Appendix 2 : Comparing GPGP and OFK}

Countries are indexed by $\mathrm{i} \in I$.

The cost of reductions of emissions of amount $q_{i}$, measured from a Business As Usual (BAU) basis are associated with a convex cost function $C_{i}$, defined for every $q_{i}$.

The utility generated in country $i$, from total reductions $Q$, is $U_{i}(Q)$, i.e $U_{i}\left(Q_{-i}+q_{i}\right)$, with $Q_{-i}=\sum_{j \neq i} q_{j}$. The function $U$ is concave.

There are two groups of countries, let us say Annex B, $(i \in B)$, where $d U_{i} / d Q>$ 0 ,in the whole range under consideration and non Annex B countries $(i \in N B)$ that are supposed to be associated with $d U_{i} / d Q=0, \forall Q$ i.e $U_{i}=C s t e(=0)$,i.e to be unconcerned ${ }^{21}$ by the policy.

We will define formally :

Definition 6. A non-cooperative Nash equilibrium, as a set of abatment levels $\overline{q_{i}}$ s.t :

$\overline{q_{i}}$ is a solution of the problem : $\operatorname{Max}\left\{U_{i}\left(\sum_{j \neq i} \overline{q_{j}}+q_{i}\right)-C_{i}\left(q_{i}\right)\right\}$.

Note that in a Nash equilibrium :

$\overline{q_{i}}>0, i \in B, \overline{q_{i}}=0, \forall i \in N B$

Note also that existence of a Nash equilibrium, follows from very weak assumptions $^{22}$. The uniqueness problem is apparently more complex : however, as it is formally analogous to the problem of existence in a Cournot oligopoly when firms have a concave maximand, we can import classical results from oligopoly theory :

\footnotetext{
${ }^{21}$ This is a too extreme assumption but a reasonable modelling option to separate B and non B countries.

${ }^{22}$ For example from the strict concavity of cost or the boundedness of the utility function.
} 
Proposition 7. - When all B-countries are similar,there exists an equilibrium .

- If the utility function is concave, there exists a unique equilibrium.

Proof. (Sketch) For part a) it is enough to note the formal similarity of our problem and of a Cournot problem. Check that, as in a Cournot problem discontinuities of best reply are upwards, and transpose the proof of BamonFraysse(1985) or Novshek (1985).

For part b), it is enough to check the continuity of best reply functions and to show that the Selten trick applies. As the Selten trick will be explained later, we advise the reader to check our suggestion after further reading.

Now we might assume, with the slight loss of generality suggested above, that the equilibrium is unique and without loss of generality that $\overline{q_{i}}=0, \forall i$. (This implies however to redefine $C_{i}\left(q_{i}\right), i \in B$, )

Let us now consider a GPGP equilibrium, associated with fixed contributions $\overline{T_{i}} . i \in B$ (i.e we assume that $B$-countries contribute the - now exogenously - fixed amount $\left.\overline{T_{i}}\right)$

Definition 8. A GPGP equilibrium, associated with fixed contributions $\overline{T_{i}}, i \in B$, consists of abatments $\left(q_{i}^{*} \geq \overline{\overline{q_{i}}}, i \in B, q_{i}^{*}>0, i \in N B\right)$, a positive "carbon price" $t^{*}$, such that :

1. $q_{i}^{*}$ is a solution of $\operatorname{Max}\left\{U_{i}\left(Q_{-i}^{*}+q_{i}\right)+t^{*}\left(q_{i}-\overline{\overline{q_{i}}}\right)-C_{i}\left(q_{i}\right)\right\}, q_{i} \geq \overline{\overline{q_{i}}}, i \in B$, with $Q_{-i}^{*}=\sum_{j \neq i} q_{j}^{*}$

2. $q_{i}^{*}$ is a solution of $\operatorname{Max}\left\{t^{*} q_{i}-C_{i}\left(q_{i}\right)\right\}, i \in N B$.

3. $\sum_{i \in B} t^{*}\left(q_{i}^{*}-\overline{\overline{q_{i}}}\right)+\sum_{i \in N B} t^{*} q_{i}^{*}=\sum_{i \in B} \overline{T_{i}}$

The GPGB equilibrium is said to be individually rational iif

4. : $\left\{U_{i}\left(Q^{*}\right)+t^{*}\left(q_{i}^{*}-\overline{\overline{q_{i}}}\right)-C_{i}\left(q_{i}^{*}\right)-\bar{T}_{i}\right\}>U_{i}\left(\sum_{i} \overline{q_{i}}\right)-C_{i}\left(\overline{q_{i}}\right)$, with $Q^{*}=\sum_{i} q_{i}^{*}, i \in$ $B$

The definition is commented in Section 2-2-1.

The existence problem is again not straigthforward. We shall prove : 
Proposition 9. If the utility function is concave, there exists for fixed normalized $\overline{T_{i}}$, a unique GPGP equilibrium.

Proof. The proof has several steps.

1- We first define a pseudo t-GPGP equilibrium, the fixed "carbon price $t$ being positive, as consisting of abatments $\left(q_{i}^{\circ}>0, i \in B \cup N B\right)$, , such that :

a) $q_{i}^{\circ}$ is a solution of $\operatorname{Max}\left\{U_{i}\left(Q_{-i}^{\circ}+q_{i}\right)+t q_{i}-C_{i}\left(q_{i}\right)\right\}, i \in B$, with $Q_{-i}^{\circ}=\sum_{j \neq i} q_{j}^{\circ}$

b) $q_{i}^{\circ}$ is a solution of $\operatorname{Max}\left\{t q_{i}-C_{i}\left(q_{i}\right)\right\}, i \in N B$.

We call $r_{i}\left(Q_{-i}, t\right)$ the best reply function, in problem a) (clearly continuous in $Q_{-i}$ and $\left.t\right)$ and write (Selten's trick) : $\left.f_{i}(Q, t)=r_{i}\left(Q-f_{i}(Q, t), t\right): f_{i}(Q, t)\right)$ is the pseudo best reply of $i$ to the total level of public good $Q$, for fixed $t$.

For $i \in N B$, we have the best reply $R_{i}(t)$, independant of $Q_{-i}$.

2- We then prove the existence and uniqueness of a pseudo t-GPGP equilibrium.

Such an equilibrium is now associated with $Q^{0}(t)$ s.t :

$Q^{0}(t)=\sum_{i \in B} f_{i}\left(Q^{0}(t), t\right)+\sum_{i \in N B} R_{i}(t)$

We then show that $d f_{i} / d Q=\frac{d r_{i} / d Q_{-i}}{1+d r_{i} / d Q_{-i}}$ and from the inspection and differentiation of the first order conditions of problem a) (left to the reader) that the denominator is positive and the numerator negative. The left-hand side of the equilibrium equation is at fixed $t$, decreasing in $Q$.

Equilibrium and uniqueness follows.

3-We now prove, as the left-hand side is increasing in t, (and equal zero (Nash) when $t=0$, that $Q^{0}(t)$ is increasing in $t$.

It follows that $t Q^{0}(t)$ is increasing in $t$, starting from zero and going to infinity. It necessarily reaches $\sum \overline{T_{i}}$, at some and unique $t^{*}$. QED.

A corollary obtains :

Corollary 10. The equilibrium carbon price of a GPGP equilibrium is increasing with $\sum \overline{T_{i}}$.

Now

Definition 11. a Flexible Kyoto equilibrium consists of (exogenously given) quotas $\widehat{q}_{i}, i \in B$, abatments $\left(q_{i}^{* \prime}\right), i \in B$, a positive "carbon price" $t^{*}$, such that :

1. $q_{i}^{* \prime}$ is a solution of $\operatorname{Max}\left\{t^{*}\left(q_{i}-\widehat{q}_{i}\right)-C_{i}\left(q_{i}\right)\right\}, i \in B$,

2. $\sum_{i \in B} q_{i}^{* \prime}=\sum_{i \in B} \widehat{q_{i}}=Q^{* \prime}$

It is individually viable if 


$$
\text { 3. } \left.\left\{U_{i}\left(Q^{* \prime}\right)\right)+t^{*}\left(q_{i}^{* \prime}-\widehat{q}_{i}\right)-C_{i}\left(q_{i}^{* \prime}\right)\right\}>U_{i}\left(\sum_{i} \overline{q_{i}}\right)-C_{i}\left(\overline{q_{i}}\right) \text {. }
$$

In a flexible Kyoto equilibrium, the sum of abatments equals the sum of initial quotas, (NB-countries are not concerned), and is taken as given in the individual optimisation that takes into account the cost and the market price of abatments (1). But quotas are traded. Here, the equilibrium is unique (the demand for abatment is an increasing function of $t$.) and Pareto superior ${ }^{23}$ to a "rigid" Kyoto equilibrium, without trade of the quotas, and hence individually viable if the former is.

Note that the real Kyoto arrangement is formally a Flexible Kyoto arrangement in which the quotas are fixed from the consideration of a basis year

Now

Definition 12. an Open Flexible Kyoto equilibrium consists of (exogenous) quotas $\widehat{q}_{i}, i \in B$, abatments $\left(q_{i}^{* *}\right), i \in I$, a positive "carbon price" $t^{* *}$, such that :

1. $q_{i}^{* *}$ is a solution of $\operatorname{Max}\left\{t^{* *}\left(q_{i}-\widehat{q_{i}}\right)-C_{i}\left(q_{i}\right)\right\}, i \in B$,

2. $q_{i}^{* *}$ is a solution of $\operatorname{Max}\left\{t^{* *} q_{i}-C_{i}\left(q_{i}\right)\right\}, i \in N B$,

3. $Q^{* *}=\sum_{i} q_{i}^{* *}=\sum_{i \in B} q_{i}^{* *}+\sum_{i \in N B} q_{i}^{* *}=\sum_{i \in B} \widehat{q}_{i}$

4. It is individually viable if $\forall i \in B:\left\{U_{i}\left(Q^{* *}\right)+t^{* *}\left(q_{i}^{* *}-\widehat{q}_{i}\right)-C_{i}\left(q_{i}^{* *}\right)\right\}>$ $U_{i}\left(\sum_{i} \overline{q_{i}}\right)-C_{i}\left(\overline{q_{i}}\right)$, with $Q^{* *}=\sum_{i} q_{i}^{* *}$.

In an Open Flexible Equilibrium, the environnemental performance is still given by the quotas of the Annex B countries, but non Annex B, which are given non binding commitments participate, and benefit from participation while decreasing the cost of Annex B countries. It is left to the reader to show that a OFK equilibrium exists is unique and involves a Pareto-improvmeent when compared with the FK equillibrium with the same $\widehat{q}_{i}$.

Our next " theorem" stresses less obvious comparative features :

1. Proposition 13. 1. Proposition 14. Given an Open Flexible Kyoto Equilibrium, there exists a family of GPGP, all with the same total contribution $\sum \overline{F_{i}}$ of Annex $B$ countries, inducing the same total abatment. In the change, the welfare level of non-Annex $B$ countries is lower, when the change of welfare of Annex $B$ countries is ambiguous. However, it is positive under the conditions set in Corollary 7, so that particpation constraints are met in GPGP if they are in an OFK.

\footnotetext{
${ }^{23}$ This is straigthforward.
} 
2. Given a GPGP equilibrium, there exists a family of OFK equilibria, each of one having the same level of total abatment. In the change the welfare level of non-Annex $B$ countries is higher.

\section{Proof.}

- Part 1)Take an OFK equilibrium, $t^{* *}, q_{i}^{* *}$.

With previous notation, it can be written :

$\sum_{i \in I} R_{i}\left(t^{* *}\right)=\sum_{i \in I} q_{i}^{* *}=\sum_{i \in B} \widehat{q}_{i}=\widehat{Q}$.

Consider the (unique) $t^{* *}-$ GPGP equilibrium, and write its global abatment

$Q^{*}=\sum_{i \in B} f_{i}\left(Q^{*}, t^{* *}\right)+\sum_{i \in N B} R_{i}\left(t^{* *}\right)=\sum_{i \in B} R_{i}\left(t^{* *}+a_{i}^{*}\right)+\sum_{i \in N B} R_{i}\left(t^{* *}\right)$, with $a_{i}^{*}=\left(\partial U_{i} / \partial Q\right)_{(Q *)}$

It is straightforward that $Q^{*}>\widehat{Q}$.

Hence, from the previous corollary, there exists a $t^{\prime}$ GPGP equilibrium, $t^{\prime}<t^{* *}$, with global abatment $\widehat{Q}$. so that the welfare of non-Annex B countries is lowered.

We have : $\sum_{i \in B} R_{i}\left(t^{\prime}+a_{i}\right)+\sum_{i \in N B} R_{i}\left(t^{\prime}\right)=\widehat{Q}$, with $a_{i}=\left(\partial U_{i} / \partial Q\right)_{(\widehat{Q})}$

The difference of welfare of one AnnexB country, measured in "money", is :

$t^{\prime} R_{i}\left(t^{\prime}+a_{i}\right)-t^{* *}\left(R_{i}\left(t^{* *}\right)-\widehat{q}_{i}\right)-C_{i}\left(R_{i}\left(t^{\prime}+a_{i}\right)\right)+C_{i}\left(R_{i}\left(t^{* *}\right)\right.$.

i.e this country would be equally well off, if it were subject to a positive $T_{i}$ transfer such that :

$t^{\prime} R_{i}\left(t^{\prime}+a_{i}\right)-t^{* *}\left(R_{i}\left(t^{* *}\right)-\widehat{q}_{i}\right)-C_{i}\left(R_{i}\left(t^{\prime}+a_{i}\right)+C_{i}\left(R_{i}\left(t^{* *}\right)\right)=T_{i}\right.$

The sum of the left hand side over $i \in B$, is, from elementary computation :

$t^{\prime} \sum_{i \in B} R_{i}\left(t^{\prime}+a_{i}\right)+t^{* *} \sum_{i \in N B} R_{i}\left(t^{* *}\right)+\sum_{i \in B} C_{i}\left(R_{i}\left(t^{* *}\right)\right)-\sum_{i \in B} C_{i}\left(R_{i}\left(t^{\prime}+a_{i}\right)\right.$

The question is whether the transfers can finance the abatment payment, whose need is :

$\sum_{i \in B} t^{\prime} R_{i}\left(t^{\prime}+a_{i}\right)+\sum_{i \in N B} t^{\prime} R_{i}\left(t^{\prime}\right)$.

Hence, one has to compare :

$\sum_{i \in N B} t^{\prime} R_{i}\left(t^{\prime}\right)$ and $t^{* *} \sum_{i \in N B} R_{i}\left(t^{* *}\right)+\sum_{i \in B}\left(C_{i}\left(R_{i}\left(t^{* *}\right)-C_{i}\left(R_{i}\left(t^{\prime}+a_{i}\right)\right)\right.\right.$

In the the case where :

$\sum_{i \in N B}\left(t^{* *} R_{i}\left(t^{* *}\right)-\left(t^{\prime} R_{i}\left(t^{\prime}\right)\right)>\sum_{i \in B}\left(C_{i}\left(R_{i}\left(t^{\prime}+a_{i}\right)\right)-\left(C_{i}\left(R_{i}\left(t^{* *}\right)\right)\right.\right.\right.$

there exists a GPGP equilibrium where Annex B countries are better off.

The right hand side equals :

$\sum_{i \in N B}\left(t^{* *}-t^{\prime}\right) R_{i}\left(t^{* *}\right)+t^{\prime}\left(R_{i}\left(t^{* *}\right)-R_{i}\left(t^{\prime}\right)\right)$

The left-hand side is smaller than :

$\left.\sum_{i \in B}\left(t^{\prime}+a_{i}\right)\left[R_{i}\left(t^{\prime}+a_{i}\right)\right)-R_{i}\left(t^{* *}\right)\right]$ 
Hence, a sufficient condition for the property is having :

$\left.\sum_{i \in N B}\left(t^{* *}-t^{\prime}\right) R_{i}\left(t^{* *}\right)+t^{\prime}\left(R_{i}\left(t^{* *}\right)-R_{i}\left(t^{\prime}\right)\right)>\sum_{i \in B}\left(t^{\prime}+a_{i}\right)\left[R_{i}\left(t^{\prime}+a_{i}\right)\right)-R_{i}\left(t^{* *}\right)\right]$

i.e , taking into account the fact that the total abatment is the same in both situations :

$$
\left.\sum_{i \in N B}\left(t^{* *}-t^{\prime}\right) R_{i}\left(t^{* *}\right)>\sum_{i \in B} a_{i}\left[R_{i}\left(t^{\prime}+a_{i}\right)\right)-R_{i}\left(t^{* *}\right)\right]
$$

Although in general this inequality may not be satisfied, it is plausible that it is.

- Part 2)

Let a GPGP equilibrium associated with $t^{*}$.

In any OFK associated with $t^{*}$, with straigthforward notation :

$\sum_{i \in B \cup N B} R_{i}\left(t^{*}\right)<\sum_{i \in B} R_{i}\left(t^{*}+a_{i}^{*}\right)+\sum_{i \in N B} R_{i}\left(t^{*}\right)$,

i.e the global abatment is smaller. However, one can choose $t^{* *}>t^{*}$ such that :

$\sum_{i \in B \cup N B} R_{i}\left(t^{* *}\right)=\sum_{i \in B} R_{i}\left(t^{*}+a_{i}^{*}\right)+\sum_{i \in N B} R_{i}\left(t^{*}\right)$,

Conclusion follows easily. QED

The question of the difference of welfare for Annex B countries for GPGP and OFK equilibria with the same level of abatment can easily be settled in some special cases. The next statement is quoted as a corollary, since it follows easily from an inequality derived in the previous proof.

Corollary 15. If all $m+n$ countries have a similar quadratic cost function $C_{i}(q)=$ $q^{2}$, if all $m$ AnnexB countries have similar preferences,

Then in the above proposition part 1, all Annex B countries are better off, as soon as the carbon price in the initial OFK equilibrium is not smaller than the marginal willingness to pay of a single Annex $B$ country..

Proof. In the above case, the above OFK and GPGP equilibria are such that

$(m+n)\left(t^{* *}\right)=m\left[t^{\prime}+a\right]+n\left[t^{\prime}\right]$

It follows that : $\left(t^{* *}-t^{\prime}\right)=[m /(m+n)] a$

The inequality :

$\left.\sum_{i \in N B}\left(t^{* *}-t^{\prime}\right) R_{i}\left(t^{* *}\right)>\sum_{i \in B} a_{i}\left[R_{i}\left(t^{\prime}+a_{i}\right)\right)-R_{i}\left(t^{* *}\right)\right]$

becomes : $\sum_{i \in N B}\left(t^{* *}-t^{\prime}\right) t^{* *}+\sum_{i \in B}\left(t^{* *}-t^{\prime}\right) a>m a^{2}$ 
$[m n /(m+n)] a t^{* *}+\left[m^{2} /(m+n)\right] a^{2}>m a^{2}$

Which holds true when $t^{* *}>a$.

QED.

\section{Appendix 3 : GPGP, between a "price" and a "quantity" policy.}

Where does stand GPGP in the standard price versus quantity debate ?

Here is a diagrammatic sketch, figure 2, of the formal argument, that refers to the standard Weitzman' framework :

On the horizontal axis is the abatment quantity, on the horizontal axis is a cost or benefit measure. I assume that, conformably to the conventional view of the greenhouse effect, the benefit curve is almost horizontal. Cost is unknown and there are a priori two different marginal cost curves.

\section{Figure 2.}

The shaded area visualizes the welfare loss due to an (optimal) quantity policy, assuming that the price policy is exactly optimal, i.e the benefit curve is exactly horizontal.

GPGP makes the spending constant and the abatments obtained as suggested on the diagram for an (approximately) optimal GPGP.

GPGP appears then as intermediate between the price policy and the quantity policy.

\section{Appendix 4 : GPGPPT and "Tax harmonisation"}

We provide here in the simple model of appendix 2, a definition of GPGP Plus Taxation equilibrium and of an harmonised tax equilibrium.

Definition 16. A GPGPPT equilibrium, associated contributions $\overline{T_{i}} . i \in B$, consists of abatments $\left(q_{i}^{*} \geq \overline{\overline{q_{i}}}, i \in B, q_{i}^{*}>0, i \in N B\right)$, a positive "carbon price" $t^{*}$, such that:

1. $q_{i}^{*}$ is a solution of $\operatorname{Max}\left\{U_{i}\left(Q_{-i}^{*}+q_{i}\right)+t^{*}\left(q_{i}-\overline{\overline{q_{i}}}\right)-C_{i}\left(q_{i}\right)\right\}, q_{i} \geq-\overline{\overline{q_{i}}}, i \in B$, with $Q_{-i}^{*}=\sum_{j \neq i} q_{j}^{*}$ 
2. $q_{i}^{*}$ is a solution of $\operatorname{Max}\left\{t^{*} q_{i}-C_{i}\left(q_{i}\right)\right\}, i \in N B$.

3. $\overline{T_{i}}=\bar{t} E_{i}^{-1}+e_{i}$,where $\bar{t}$ is a tax rate and $E_{i}^{-1}$ is the emissions of country $i$ at period -1 .

4. $\sum_{i \in B} t^{*}\left(q_{i}^{*}-\overline{\overline{q_{i}}}\right)+\sum_{i \in N B} t^{*} q_{i}^{*}=\sum_{i \in B} \overline{T_{i}}$

It is easy to check that choosing $\left.\bar{t}=\operatorname{Min} \overline{\left(T_{i}\right.} / E_{i}^{-1}\right)$,allows to adjust the GPGP contributions to any a priori given profile of contributions.

Definition 17. An harmonised Tax equilibrium consists of abatments $\left(q_{i}^{00}\right), i \in I$, a positive "carbon tax" $t^{00}$, such that :

1. $q_{i}^{00}$ is a solution of $\operatorname{Min}\left\{t^{00}\left(q_{i}\right)-C_{i}\left(q_{i}\right)\right\}, i \in I$,

2. $Q^{00}=\sum_{i} q_{i}^{00}=\sum_{i} R_{i}\left(t^{00}\right)$

3. The welfare of country $i$ is $\left\{U_{i}\left(Q^{00}\right)-C_{i}\left(R_{i}\left(t^{00}\right)\right)\right\}$

The reader will note that a tax equilibrium is an OFK with $\widehat{q}_{i}=R_{i}\left(t^{00}\right)$

\section{Appendix 5 : Tax incidence for an exhaustible resource.}

We refer to the model sketched in the text : pure competition, perfecf foresight, constant discount rate, time invariant demand function, and different assumptions on the boundary behaviour of prices

- Case 1 :

The resource is exhausted at time $T$, before it gets a substitute :

The producer price is denoted $p$, the total price is denoted $P$, the difference is the $\operatorname{tax} \tau$.

Necessarily : $p(t)=p(0) \exp (r t), P(t)=p(t)+\tau(t)$.

An increase of a tax, from $\tau(t)$, to $\tau^{\prime}(t)=\tau(t)+\epsilon, \bar{t} \leq t \leq \bar{t}+\epsilon,(\tau(t)=$ $\tau^{\prime}(t)$ elsewhere) reduces the producer price from $p(0) \exp (r t)$ to $p^{\prime}(0) \exp (r t)$ s.t : $\int_{0}^{T} D\left(p^{\prime}(0) \exp (r t)+\tau^{\prime}(t)\right) d t=\int_{0}^{T} D(p(0) \exp (r t)+\tau(t)) d t$.

A temporary increase in the tax depress the producer price all over the period and depress the total price all over the period, but at the time the tax is increased. 
- Case 2 :

There is a (perfect) substitute at time $T^{\prime}$, of price $v$. After time $T$, the final price of the resource is $v$, and the demand is shared between the resource and its substitute in the proportion $\alpha$ and $1-\alpha$. The resource is exhausted at time $T$.

Necessarily : $p(t)=p(0) \exp (r t), P(t)=p(t)+\tau(t), P(t)=v, t \geq T^{\prime}$.

A temporary increase of a tax, from $\tau(t)$, to $\tau^{\prime}(t)=\tau(t)+\epsilon, \bar{t} \leq t \leq \bar{t}+\epsilon<T^{\prime}$, reduces the producer price from $p(0) \exp (r t)$ to $p^{\prime}(0) \exp (r t)$ and increase the exhaustion time to $\bar{T}$ s.t :

$$
\begin{aligned}
& \int_{0}^{T^{\prime}} D\left(p^{\prime}(0) \exp (r t)+\tau^{\prime}(t)\right) d t+\int_{T^{\prime}}^{\bar{T}} \alpha D(v) d t=\int_{0}^{T^{\prime}} D(p(0) \exp (r t)+\tau(t)) d t .+ \\
& \int_{T^{\prime}}^{T} \alpha D(v) d t
\end{aligned}
$$

\section{Appendix 6 : Uncertainty on tax incidence : an example where a quantity policy is better than a price policy}

Here, figure 3, the price policy consists of an harmonised tax. However the effect of the harmonised tax on the carbon price is uncertain in the medium run, reflecting

- the uncertainty on the future tax,

- the absence of commitment,

- the difficulty of predicting the medium run reaction of producers price and the difficulties of expectational coordination ("eductive instability").

Then for a given tax decision, there are two possible values of carbon prices, one over the marginal benefit and the other one below. In the same framework as appendix 3, figure 3 visualises a case where the pure quantity policy (Kyoto like) is superior to the price policy.

Figure 3 
Figure 1:

10. Figures. 
Figure 2:

\section{Bibliography.}

Aldy, J.E., P. R. Orszag \& J. E. Stiglitz, "(2001) "Climate Change: An Agenda for Global Collective Action", Prepared for the conference on "The Timing of Climate Change Policies", Pew Center on Global Climate Change, October

Barrett, S., (2001), "Towards a Better Climate Treaty", Policy Matters 01-29, Brookings Institution, November

Bamon R. and Fraysse J (1985) "Existence of Cournot Equilibrium in large Markets", Econometrica, 53, (3), 587-597.

Bradford, D.F. (2001), "Improving on Kyoto: A No Cap but Trade Approach to Greenhouse Gas control", Princeton University, in this volume, Chapter 1 ..

Chakrovorty U, Magné B., Moreaux M, (2003) « Energy resource substitution and carbon concentration targets with non stationary needs", Leerna 31, Université Toulouse 1.

Cooper, R., (1998), "Toward a real global warming treaty", Foreign Affairs, vol. $77 \mathrm{n}^{\circ} 2$, March/April

Carraro C.(1999) "The Structure of International Agreements on Climate 
Figure 3:

Change"in C. Carraro C. (ed), International Environmental Agreements on Climate Change, Kluwer Academic Publishers, Dordrecht, NL

Chandler H. Tulkens () International Journal of Game Theory

Freixas X, Guesnerie R, et Tirole J. (1985) « Planning under incomplete information and the ratchet effect », Review of Economic Studies, LII, 173-191..

Guesnerie R. (2003) "Les enjeux économiques de l'effet de serre" in « Kyoto et l'économie de l'effet de serre", sous la direction de R. Guesnerie, La Documentation Française, Paris.

Guesnerie R ( 2004) « Calcul économique et Développement durable », Revue Economique, p.363-382.

Guesnerie R. (2005) "Assessing Rational Expectations :2- "Eductive" stability in economics, MIT Press, 453p.

Ha-Duong M, Grubb M et. Hourcade J.C, (1997) "Influence of socio-economic inertia and uncertainty on optimal CO2-emissions abatment", Nature, Vol. 390.

Hargrave, T., (1998), "US carbon emissions trading: description of an upstream approach", Centre for Clean Air Policy, Washington D.C., July

Hoel, Michael, and Larry S. Karp.(2001). "Taxes and Quotas for a Stock 
Pollutant with Multiplicative Uncertainty." Journal of Public Economics, Vol. 82, pp. $91-114$

Hoel, Michael, and Larry S. Karp. (2002)"Taxes Versus Quotas for a Stock Pollutant."Resource and Energy Economics, Vol. 24, pp. 367-384 .

Karp, Larry S., and Jiangfeng Zhang. (2005) "Regulation of Stock Externalities withCorrelated Abatement Costs." Environmental and Resource Economics, Vol. 32

No. 2 pp $273-300$.

Karp, Larry S., and David M. Newbery.(1993) "Intertemporal Consistency Issues in Depletable Resources." In Handbook of Natural Resources, Vol. III, edited by

Allen Kneese and James Sweeny. North Holland, New York, , pp. 881-930.

McKibbin, W. J. \& P. J. Wilcoxen, (2000), "Designing a Realistic Climate Change Policy that includes Developing Countries", Paper for the 2000 Conference of Economists, the Gold Coast, July 3-5

Magné B., Moreaux M. (2002) "Long run energy trajectories : assessing the nuclear option in response to global warming", Leerna DP 0226101, Université Toulouse 1.

Neary P (1999) "International trade and the environnement : theoretical and policy linkages", mimeo;

Newell, R.G. \& W.A. Pizer, (2000), "Regulating Stock Externalities Under Uncertainty", Discussion Paper 99-10, Resources for the Future, Washington DC, February.

Nordhaus, W.D, (2002), "After Kyoto: Alternative Mechanisms to Control Global Warming", Paper prepared for the meetings of the American Economic Association and the Association of.IEA/SLT(2002)28

Novshek W. (1985) "On the existence of Cournot equilibrium", Review of Economic Studies 52, 85-88.

Philibert, C. (2000). "How could emissions trading benefit developing countries." Energy Policy, volume 28, n¹3.

Philibert, C., and J. Pershing. (2001). "Des objectifs climatiques pour tous les pays : les options." Revue de l'énergie 524.

Pizer, W.A., (2001), "Combining Price and Quantity Control to Mitigate Global Climate Change", Journal of Public Economics, 85,(3), 409-434.

Victor, D., 2001, "The Collapse of the Kyoto Protocol and the Struggle to Slow Global Warming", Princeton University Press, Princeton, NJ.

Rieu J.(2002) "Politiques nationales de lutte contre le changement climatique 
et réglementation de la concurrence : le cas de la fiscalité", mimeo.

Tulkens H. (2005) « Stability issues and climate related dynamic externalities $\gg$, this conference.

Weitzman, M. L., (1974), "Prices vs. Quantities", Review of Economic Studies, vol.41, October. 